DE

M E D I C I N A

T R O P I C A L

$\mathrm{DE}$

SÃO PAULO

JOURNAL OF THE SÃO PAULO INSTITUTE OF TROPICAL MEDICINE

${ }^{1}$ Islamic University of Madinah, Madinah, Saudi Arabia

${ }^{2}$ King Fahd University of Petroleum and Minerals, Dhahran, Saudi Arabia

Correspondence to: Mohammad A. R. Abdeen

Islamic University of Madinah, Prince Naif Ibn Abdulaziz, Al Jamiah, Medina, 42351, Madinah, 86421, Saudi Arabia

E-mail: mabdeen@iu.edu.sa

Received: 8 March 2021

Accepted: 14 May 2021

\section{An approximate analytical formula for estimating the weight of factors affecting the spread of COVID-19: a case study of the first wave}

Mohammad A. R. Abdeen ${ }^{1}$, Tarek R. Sheltami², Ibrahim A. Nemer ${ }^{2}$

\section{ABSTRACT}

COVID-19 pandemic has changed the way we live our lives for the foreseen future. To date, there have been over 113 million reported cases and 2.5 million deaths worldwide. Many studies investigated the factors affecting the number of daily cases such as weather conditions, lockdown duration and other factors. In this study, we propose a COVID-19 analytical formula for factors contributing to the number of the new coronavirus daily cases. We have also calculated values of relative weights of those factors. We focus on the first wave data that are publically available. Seven countries were considered including the UK, Italy, Spain, Canada, South Korea, Germany and France. We considered the following factors: temperature, humidity, government expenditure, lockdown hours and the number of daily tests for COVID-19 performed. The weights were calculated based on the hypothesis that a high correlation between recorded data of a given pair of countries implies a high correlation of the pair's COVID-19 proposed analytical formula. The factors are calculated using the brute-force technique. Our results showed that in five out of the seven countries; temperature, humidity, and lockdown duration were the most dominant with values of $26 \%$, $32 \%$ and $38 \%$, respectively. In other countries, however, humidity, government expenditure and the daily performed tests for COVID-19 were the most effective factors, with relative values of $35 \%, 26 \%$, and $28 \%$.

KEYWORDS: COVID-19. Correlation coefficient. Environmental factors. Lockdown. Inductive analysis.

\section{INTRODUCTION}

The world faced a new challenge when it was hit by one of the worst pandemics in recent history, COVID-19. Coronaviruses are a large family of viruses that cause respiratory diseases with symptoms ranging from common cold to more severe diseases such as the Severe Acute Respiratory Syndrome (SARS-CoV). The novel coronavirus has not been previously found in humans and causes an illness called COVID-19. The first case of COVID-19 was reported in December 2019 in Wuhan province, China. To date, over 40 million people have contracted the virus and the death toll is over 1.1 million people ${ }^{1}$. The spread of the coronavirus has peaked in many countries in the months of March and April ${ }^{2}$. According to the World Health Organization (WHO) a vaccine might be available in 12 to 18 months $^{3}$.

The damage caused by the pandemic has been multifaceted. Its effects were sociological, physiological, political and economic. People have changed their daily routines to avoid being infected with the virus and apply social distancing rules. Many countries have set medium-to-severe lockdown policies and quarantine regions 
to isolate the virus and cripple its spread. On the other hand, the economic impact of the closures and lockdown measures implemented by the majority of the countries have had a damaging effect on the economy and the living standards. According to a report by Bloomberg, the cost of COVID-19 can be as much as $\$ 2.7$ trillion $^{4}$. Therefore, world countries have been sparing no effort in trying to evade this virus and also minimize the economic impact resulting from the curfew hours and lockdown regions.

Despite the relative cessation in the spread of the novel coronavirus in many countries during the third quarter of 2020 , it started to show signs of a second wave especially in Europe. Many researches have attempted to produce a vaccine with only a few approved to date. On a different front, other solutions are being proposed in the form of producing models for the purpose of understanding the characteristics of the virus and learning its curves to transfer this knowledge to other regions or may be to avert a second and a third wave ${ }^{5}$.

Some organizations have made the data on the daily cases and daily deaths of countries or specific countries (such as the US) available through an online dashboard ${ }^{1,6,7}$. These data has proven very useful in understanding the past and current trends and in attempting to uncover their relationships with factors that might affect them, such as the weather conditions, the government policies like the curfew hours, population densities, the expenditure in the healthcare sector and other conditions.

On top of that, the measures taken by various countries are different in time and space and their effects are manifested in the reported curves of daily infections and deaths. An interesting and urgent question is how to mine the relationship between those curves and the other factors affecting the spread of the coronavirus including the shutdown/curfew hours.

The contributions of this paper are: (1) We proposed a generic analytical and quantitative methodology using the correlation function between reported coronavirus data (daily cases and daily deaths) to analyze five main factors affecting the spread of the coronavirus (temperature, humidity, lockdown hours, expenditure and daily tests performed to COVID-19); (2) We obtain percentage values of the relative weights of the factors affecting the spread of the virus; (3) We uncovered similarities of countries considered in this study thereby lessons can be learned from countries in the same group.

The rest of the manuscript consists of four sections. Section II introduces the related studies. Section III introduces the methodology and the formulation of the inductive formula. Section IV presents the results of our proposed approach. Section V discusses the results and introduces some reflections. Section VI summarizes the research achievement and suggests futurestudies.

\section{RELATED STUDIES}

Many reports in the literature exist regarding the relationship between the spread of an infectious disease and environmental factors ${ }^{8-10}$. Pica and Bouvier ${ }^{8}$ presented a study on environmental factors affecting the spread of respiratory diseases such as influenza and SARS coronavirus. They reported that the transmission increases with lower temperatures and lower relative humidity index (20\%). Another recent study ${ }^{11}$ investigated the effects of the economic and social factors on the spread of the novel coronavirus (COVID-19) in Thailand. They found a positive and relatively high correlation between the number of tourists and the social distancing policies. The study was rather qualitative and empirical.

Rashed et al..$^{9}$ presented a study performed in Japan to investigate the effects of environmental factors such as temperature and humidity on the spread and the decay duration of the coronavirus. They found a positive correlation between the decay period and the temperature and relative high humidity values. Another study showed the effect of environmental factors on the spread of the COVID-19 in Turkey $^{10}$. The author found a positive correlation between the number of reported daily cases in 10 Turkish cities with the temperature and the average wind speed.

The previous studies despite showing results suggesting positive correlations with environmental conditions such as temperature, humidity, wind speed and UV radiation, they were qualitative studies and came short of presenting the relative weights of those factors on the spread of the novel coronavirus. Mathematical models have been proposed in the literature for the modeling of the infection dynamics. As an example, Tariq et al. ${ }^{12}$ studied the infection dynamics of Ebola in Democratic Republic of Congo (DRC) based on estimating infection reproduction number R0 from May 2018-January 2019 using the Renewal Equation Method $(\mathrm{REM})^{13}$. The authors presented an estimate of the potential impact of reporting delays on distorting the epidemic incidence pattern misrepresented.

Recent studies have addressed the modeling on the spread of the novel coronavirus. In Jia et al. ${ }^{14}$ three mathematical models, a logistic model, the Bertalanffy model, and the Gompertz model, have been investigated. The epidemic trends of SARS were first fitted and analyzed in order to prove the validity of the existing mathematical models. The results were then used to fit and analyze the situation of COVID-19. Liang ${ }^{15}$ executed spread parametric 
analysis of the three pneumonia: COVID-19, SARS and MERS. The authors compared the infection kinetics growth of the three outbreaks by establishing a mathematical propagation growth model. The parametric analysis results explained that the infection growth rate of COVID-19 is much higher than those of SARS and MERS, which is about twice that of the SARS and MERS. Li et al. ${ }^{16}$ analyzed data on the first 425 confirmed cases in Wuhan to determine the epidemiologic characteristics of COVID-19 based on a laboratory testing and statistical analysis approach.

Shim et al. ${ }^{17}$ analyzed the COVID-19 spread in South Korea. The authors extracted the daily confirmed cases of COVID-19 in South Korea from publicly available data sources, then by using the empirical reporting delay distribution and simulating the generalized growth model. The findings proved that implementing the social distancing measures in South Korea is related to the rapidly control of the outbreak of COVID-19. D'Arienzo and Coniglio ${ }^{18}$ investigated and assessed data derived from the early phase, in the interval between February 25-March 12, 2020, of the outbreak of COVID-19 in Italy. The authors modeled the disease spread in the top nine Italian cities by applying the well-established SIR-model. Their objective was to estimate the basic reproduction number, $R_{0}$. Their findings were that it ranged from 2.4-3.10, which agreed with other values reported in the literature. In another study in Italy, the authors investigated the challenges faced by healthcare workers and their effects on the process of dealing with the epidemic ${ }^{19}$. Their study presented statistical data on the number of deceased healthcare professionals due to COVID-19. They suggested that decision makers should propose a penal shield to maintain the front-line workers within the system to win the fight against COVID-19.

Other factors affecting the spread of the coronavirus such as the relationship with other epidemics have been reported in the literature. Huang et al..$^{20}$ presented a study that linked different regions kin which the spread of malaria is known, specifically African countries such as Nigeria, Uganda and Niger and the relatively slow and lower number of COVID-19 cases. The authors suggested a correlation between the gain of immunity due to malaria and the susceptibility to COVID-19 infection.

Other techniques such as the spatio-temporal modeling of various factors including meteorological ones have been reported in the literature ${ }^{21-23}$. Briz-Redón and SerranoAroca $^{23}$ presented a spatio-temporal modeling approach to explore the temperature effects on the spread of COVID-19 in Spain. They performed the study in 47 provinces and found a positive association between the mean temperature and the newly confirmed COVID-19 cases.

Other non-analytical approaches exit in the literature to study the factors affecting the spread of the new coronavirus. Lakshmi Priyadarsini and Suresh ${ }^{24}$ have used the Total Interpretive Structural Modeling (TISM) methodology to identify and rank the factors affecting the spread of the coronavirus. Their approach is non analytical, and the factors were identified using "expert opinions" of 27 professors, scientists and researchers. They identified 15 factors thereafter divided into four different groups (called zones): the autonomous factors, the dependent factors, the linkage factors and the driving factors. Three of the 15 factors (the host defense potential, the underlying health condition, and the virulence ofSARS-CoV-2 ) were identified as dependent factors. The driving factors were defined as the ones that have a strong driving power.

In the study presented herein, we have considered the driving factors presented by Lakshmi Priyadarsini and Suresh ${ }^{24}$. Those factors are: the social distancing (a.k.a. lockdown), the air temperature, humidity and the population density (data were normalized). Other authors have reported the effect of non-meteorological factors such as expenditure and daily tests performed for COVID-19. Those factors have also been considered in this study due to their significance ${ }^{25,26}$.

\section{METHODOLOGY}

The proposed approach in this study is about leveraging the authentic recorded daily infected cases as well as daily deaths of a given pair of countries. We selected seven countries for our study namely, Italy, Spain, the UK, Germany, South Korea, Canada and France. Those countries were listed among the top affected by the first wave of the pandemic. For those countries, there are $7 ! /(5 !)(2 !)=21$ unrepeated pairs. For each of these pairs, a correlation value was calculated. We have set a threshold for the correlation value for which we claimed that a pair of countries is/is not correlated.

\section{Input data utilized}

In this study, we considered five factors that are believed to affect the daily cases of the new coronavirus. These factors are the weather conditions (i.e., temperature, humidity), expenditure, curfew hoursand the number of cases/deaths and the number of testsfor COVID-19 performed. We have made a simplifying assumption so that we will use the average data of the two largest cities (population wise) as representative of a given country.

1) Data collection: We obtained the required datasets to perform this study from various public websites for 
the period between March 15, 2020 - June 30, 2020 as follows: Cases/deaths per day ${ }^{1}$, curfew hours and tests ${ }^{27}$, weather conditions ${ }^{28}$ and expenditure ${ }^{29}$. We basically use the average value from the collected data for each month to be the targeted value. Hence, we have four values (i.e. March, April, May, June) from the selected dataset. These values are used later on to figure out the approximated formula for each country.

2) Method of sampling and visualization: A 10-day moving average to represent the daily cases for each country based on the collected data was used ${ }^{1}$. A 10-day moving average represents a mathematical model to approximate and give a prediction/fit for a value based on averaging 10 days in this study. The formula for evaluating the moving average (MA) is as follows:

$$
M A=\frac{V_{1}+V_{2}+\ldots V_{n}}{n}
$$

where V1 represents the first value in the selected period $\mathrm{n}$. We plot the normal daily cases, 3-day moving average, 7-day moving average and 10-day moving average per population size for each country using the collected daily cases as in Figure 1.

\section{Inductive formula}

This study considers five factors that are thought to affect the spread of the coronavirus for a given country (section 3.1). The dataset of each country is represented by a set of five polynomial equations of fourth and fifth degree, depending on the input accuracy. The following are the five factors considered:

1) Temperature

2) Humidity

3) Expenditure

4) Curfew hours

5) Number of tests performed

The proposed COVID-19 inductive formula for a given country is given by:

$$
\begin{aligned}
& n e w E_{q_{\text {country }}}=\left(k_{1} \times \text { temperatureE } q\right)+\left(k_{2} \times \text { humidity } E q\right)+ \\
& \left(k_{3} \times \text { expenditure } q\right)+\left(k_{4} \times \text { curfew } E q\right)+\left(k_{5} \times \text { tests } E q\right)
\end{aligned}
$$

The problem now is finding the optimal values for the parameters $\mathrm{K}_{1}$ to $\mathrm{K}_{5}$, where:

$$
\sum_{i=1}^{5} K i=1
$$

It is worth noting that the effect of the number of tests per day performed by a given country is a second order parameter. It does not directly affect the spread of the coronavirus, but it affects the reported number of cases, which is the reason for including it as an effective factor. The purpose of COVID-19 inductive formula is to enable us to identify the most dominant factors affecting the spread of the coronavirus. The optimal values are obtained when the difference in correlation (deltaCorr) values between any pair of countries is below a predefined error value (0.01). The delta correlation is:

$$
\text { deltaCorr }=\left|\left(\frac{\text { predCorr }- \text { DSCorr }}{D S C o r r}\right) \times 100\right|
$$

We use the daily reported cases (infected and death) to obtain the equation for these counties. The overall correlation is calculated based on both, the daily infected cases and the daily deaths as they are the two most important factors to describe the state of the disease in a given country. We postulate using a complex variable to obtain the overall correlation $X+\mathrm{j} Y$ : the normalized new cases $(X)$ represents the real part and the normalized deaths cases $(Y)$ represents the imaginary part. The overall correlation coefficient based on the obtained dataset (DSCorr) of any pair of countries was obtained using the following formula:

$$
\text { DSCorr }=\frac{\left(\sqrt{(\text { Xcorr })^{2}+(\text { Ycorr })^{2}}\right)}{\sqrt{2}}
$$

The difference of correlation coefficients (deltaCorr) between the predicted and dataset-based correlation was then calculated using equation (4). The optimal K's values are obtained by minimizing the K-values ( 0 to 1 ).

\section{RESULTS}

We developed a mathematical model that combines the proposed factors affecting the spread of the coronavirus and their relative weights. We have considered seven countries that had the highest relative number of daily infections in the considered time period. These countries were the UK, Italy, Spain, Canada, South Korea, Germany and France. Weather conditions (temperature and humidity), expenditure, curfew hours and testing of COVID-19 cases were the main factors that we used to model the function based on the collected data during the period from March 15, to June 30, 2020. We investigated the functions based on two scenarios: first we assumed 

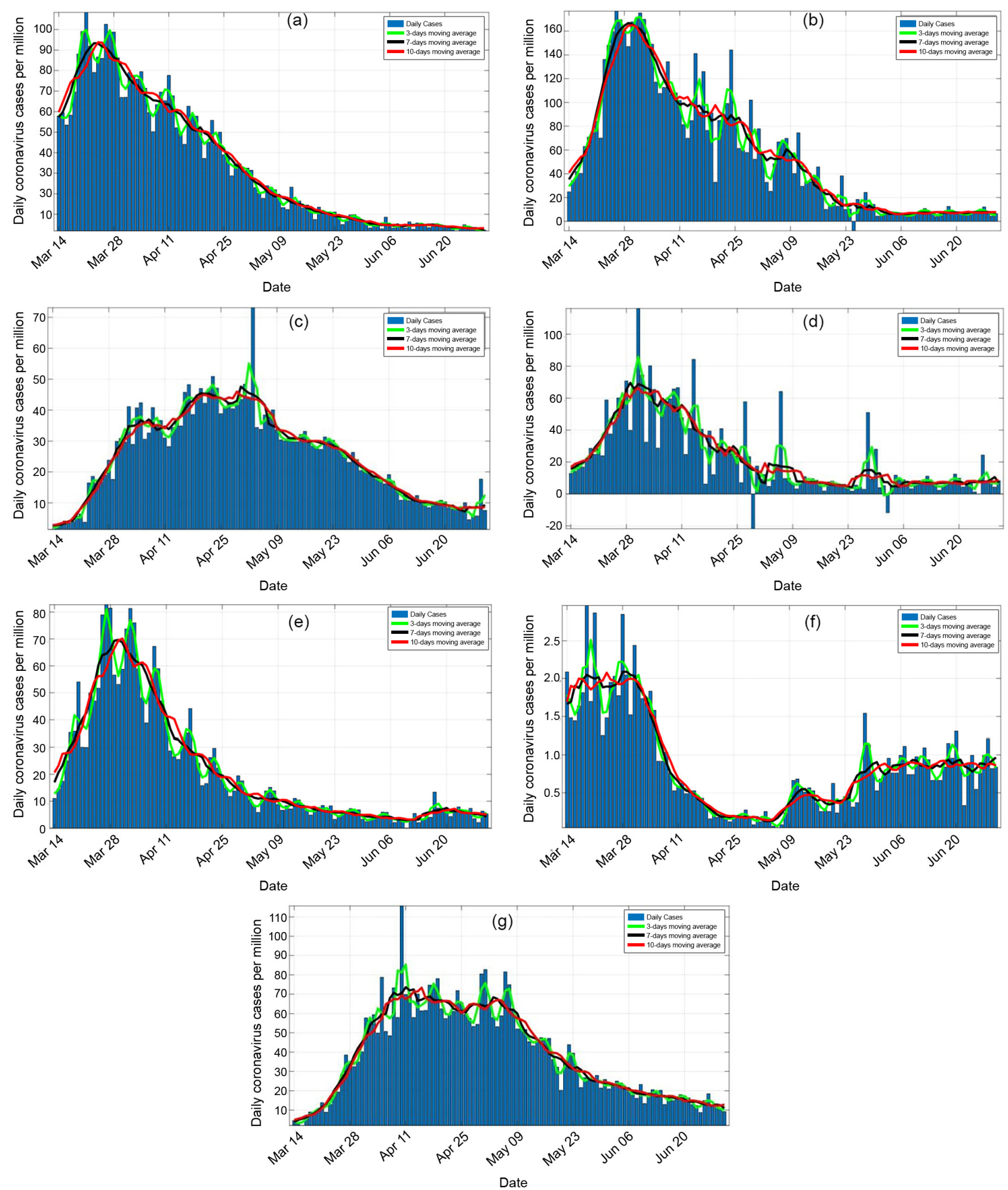

Figure 1 - Normalized new cases from March 15, 2020 to June 30, 2020: (a) Normalized new cases in Italy; (b) Normalized new cases in Spain; (c) Normalized new cases in Canada; (d) Normalized new cases in France; (e) Normalized new cases in Germany; (f) Normalized new cases in South Korea; (g) Normalized new cases in the UK.

a global function for all countries and calculated the dependency constants (K1 - K5). Next, we considered each country to be distinct and calculated the dependency constants (K's) for each country separately.

\section{Predicted set of equations}

The output of the fitting stage with minimum error for a given country (e.g. Italy) for each factor was represented 
by a nonlinear polynomial of third degree as follows:

$$
F_{\text {actor } \text { Italy }_{\text {f }}}=P 1 \times z^{3}+P 2 \times z^{2}+P 3 \times z+p 4
$$

As an example, the coefficients for temperature in Italy were given by the set $\left(p_{1}=0, p_{2}=0.004, p_{3}=-0.16, p_{4}=17.6\right.$ ) while the coefficients for humidity in Italy were given by the set $\left(p_{1}=0, p_{2}=-0.003, p_{3}=0.24, p_{4}=68.6\right)$. Other factors were calculated similarly. Figure 2 below depicts the used curves for Italy as an example.

The output values from these equations were then normalized using the mean value for each equation in order to have the same range of values. Next, we combined the five equations for each country into one equation, and the equation of Italy was as follows:
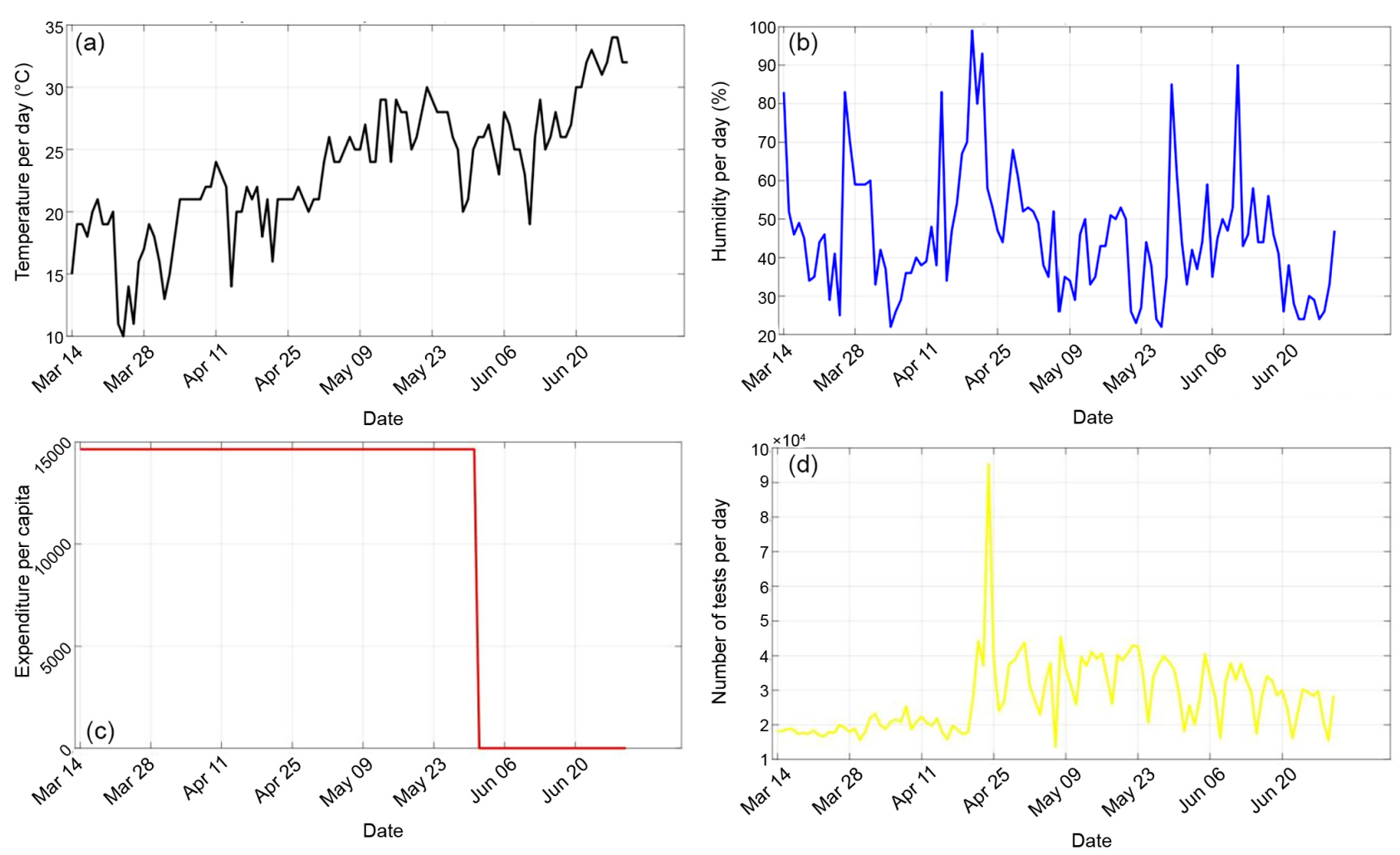

$$
\begin{aligned}
& n e w_{E_{\text {qlaty }}}=\left(k_{1} \times \text { temperature } E q\right)+\left(k_{2} \times \text { humidity } E q\right)+ \\
& \left(k_{3} \times \text { expenditure } q\right)+\left(k_{4} \times \text { curfewE } q\right)+\left(k_{5} \times \text { tests } E q\right)
\end{aligned}
$$

where $k_{1}$ to $k_{5}$ are the dependency constants for each equation. The predicted correlation (predCorr) between any two countries was found using the predicted equations based on the selected $\mathrm{K}$ values. After we define the delta correlation expression, we figure out the optimal K's based on the minimum correlation that can be reached.

\section{Delta correlation between predicted and fitted data equations}

We evaluated the delta correlation between the values based on the inductive formula and those obtained using

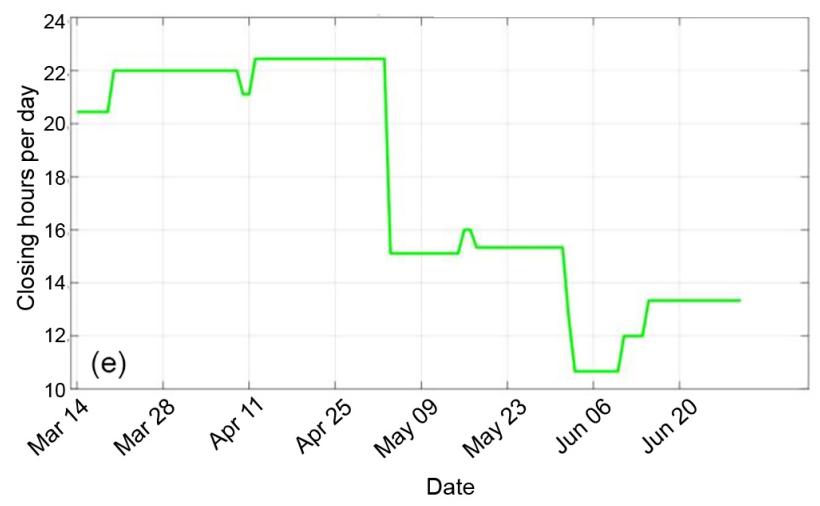

Figure 2 - The real data curves: (a)Temperature; (b) Humidity; (c) Lockdown hours; (d) Expenditure; (e) Number of COVID-19 tests in Italy, from March $14^{\text {th }}, 2020$ to June $30^{\text {th }}, 2020$. 
the reported dataset as follows:

$$
\text { deltaCorr }=\left|\left(\frac{\text { predCorr }- \text { DSCorr }}{D S C o r r}\right) \times 100\right|
$$

Two scenarios have been considered in obtaining the value of $\left(\mathrm{K}_{1}-\mathrm{K}_{5}\right)$. In the first scenario we assumed a global formula that was presumably valid for all countries. The values of K's were calculated through a brute-force approach since we could assume a discreet optimization space.

1) Objective was to minimize deltaCorr

2) Constraints were $\sum_{i=1}^{5} K_{i}=1 \& K^{\prime} s:[0,1]$

The $\mathrm{K}$-values that achieved the minimum delta values were $\left(K_{1}=0.03, K_{2}=0.78, K_{3}=0.007, K_{4}=0.17\right.$, $K_{5}=0.0008$.

Table 1 below shows the results obtained with this approach (global minimum). These results presented very poor values of deltaCorr for many pairs of countries (the deltaCorr can go over 100\%).

This finding suggested that there was no global minimum that fit all countries, and a search for a local minimum was a more viable option. A second approach was therefore presented for which values of $\mathrm{K}$ were calculated to minimize deltaCorr for each pair of countries. The values of deltaCorr were calculated using a brute force approach by iterating through all possible values of the $\mathrm{K}$ parameters with a step of 0.01 to minimize the computation time. The result is shown is Table 2.
We made an assumption that two countries were correlated if the correlation was more than approximately $60 \%$ (to be exact, $57 \%$ ). We ended up with 11 correlations, which were highlighted in orange in Table 2 and are given by the symbols V1, V3, V4, V5, V8, V9, V10, V15, V16, V18, V20.

In this scenario, we considered each country to be distinct, and evaluated the dependency constants (K's) for each pair of countries separately as shown in Table 2 .

\section{DISCUSSION}

In this section, we analyze the data shown in Table 3 and find the max frequency of occurrence for the $\mathrm{K}$ values in triplets. Possible triplets of highest $\mathrm{K}$ values for the seven countries are highlighted in green in Table 3. As shown in the table, $\mathrm{K}_{1}$ (temperature), $\mathrm{K}_{2}$ (humidity) and $\mathrm{K}_{4}$ (lockdown) were the most dominant triplets, with a frequency of occurrence of 5 out 11 . They appeared in pairs (V3, V4, V5, V10, V16) and corresponded to a group of countries including Germany, France, South Korea, Italy and Spain.

The second most dominant triplet was the $\mathrm{K}_{2}$ (humidity), $\mathrm{K}_{3}$ (expenditure), and $\mathrm{K}_{5}$ (number of tests) with a frequency of value occurrence of 2 . This triplet corresponded to the countries of Canada and the UK. It can also be shown that considering the individual frequency of each factor (by counting the frequency of each K-value given in

Table 1 - deltaCorr values for all the seven countries based on the first scenario.

\begin{tabular}{lccccccc}
\hline Country & Italy & Spain & Canada & France & Germany & S. Korea & UK \\
\hline Italy & 0 & 8.03119611 & 124.4241162 & 10.90811915 & 21.5545879 & 64.84800001 & 47.96401785 \\
Spain & 8.03119611 & 0 & 123.0584752 & 14.68718263 & 37.6048039 & 29.69210805 & 64.09843097 \\
Canada & 124.4241162 & 123.0584752 & 0 & 70.15732918 & 85.68314818 & 107.733477 & 27.76765187 \\
France & 10.90811915 & 14.68718263 & 70.15732918 & 0 & 69.48854017 & 109.6103642 & 9.012849874 \\
Germany & 21.5545879 & 37.6048039 & 85.68314818 & 69.48854017 & 0 & 76.97160353 & 59.18372077 \\
S. Korea & 64.84800001 & 29.69210805 & 107.733477 & 109.6103642 & 76.97160353 & 0 & 99.45439057 \\
UK & 47.96401785 & 64.09843097 & 9.012849874 & 9.012849874 & 59.18372077 & 99.45439057 & 0 \\
\hline
\end{tabular}

Table 2 - Delta correlation values for actual infected and death cases.

\begin{tabular}{lccccccc}
\hline Country & Italy & Spain & Canada & France & Germany & S. Korea & UK \\
\hline Italy & 1 & $\mathrm{~V} 1=0.889$ & 0.143 & $\mathrm{~V} 3=0.669$ & $\mathrm{~V} 4=0.740$ & $\mathrm{~V} 5=0.680$ & 0.394 \\
Spain & & 1 & 0.290 & $\mathrm{~V} 8=0.753$ & $\mathrm{~V} 9=0.786$ & $\mathrm{~V} 10=0.574$ & 0.537 \\
Canada & & 1 & 0.188 & 0.301 & 0.441 & $\mathrm{~V} 15=0.709$ \\
France & & & 1 & $\mathrm{~V} 16=0.776$ & 0.413 & $\mathrm{~V} 18=0.593$ \\
Germany & & & & & 1 & 0.443 & $\mathrm{~V} 20=0.641$ \\
S. Korea & & & & & & 1 & 0.354 \\
UK & & & & & & & 1 \\
\hline
\end{tabular}


Table 3 - Optimal K-values for counties with high correlation based on the second scenario.

\begin{tabular}{lcccccc}
\hline & Delta Correlation & $\mathrm{K}_{1}$ & $\mathrm{~K}_{2}$ & $\mathrm{~K}_{3}$ & $\mathrm{~K}_{4}$ & $\mathrm{~K}_{5}$ \\
\hline V1 & 0.006 & 0.075 & 0.344 & 0.293 & 0.030 & 0.257 \\
V3 & 0.009 & 0.262 & 0.320 & 0.018 & 0.395 & 0.003 \\
V4 & 0.030 & 0.197 & 0.276 & 0.086 & 0.268 & 0.171 \\
V5 & 0.000 & 0.285 & 0.232 & 0.013 & 0.327 & 0.140 \\
V8 & 0.006 & 0.013 & 0.305 & 0.165 & 0.251 & 0.264 \\
V9 & 0.008 & 0.160 & 0.054 & 0.191 & 0.212 & 0.381 \\
V10 & 0.041 & 0.340 & 0.085 & 0.069 & 0.451 & 0.053 \\
V15 & 0.013 & 0.217 & 0.235 & 0.223 & 0.172 & 0.151 \\
V16 & 0.050 & 0.192 & 0.400 & 0.132 & 0.273 & 0.002 \\
V18 & 0.045 & 0.244 & 0.217 & 0.245 & 0.236 & 0.055 \\
V20 & 0.062 & 0.031 & 0.359 & 0.236 & 0.0494 & 0.322 \\
\hline
\end{tabular}

Table 3), they will lead to the temperature, humidity and the lockdown as the most frequent factors.

To validate our findings, the correlation values of various pairs of countries have been calculated using the proposed analytical formula and the corresponding K-values. Table 4 shows the correlation values of pairs corresponding to V3 (France-Italy), V4, V5, V10, V16. These values showed a high level of correlation in almost all cases. One exception was the case of V10 (Spain - South Korea) which might indicate a variation in the government-imposed curfew policies between the two countries.

Table 4 - Values of correlation between the actual temperature, humidity and lockdown.

\begin{tabular}{lccc}
\hline & Temperature & Humidity & Lockdown \\
\hline V3 & 0.95 & 0.95 & 0.63 \\
V4 & 0.96 & 0.96 & 0.60 \\
V5 & 0.91 & 0.88 & 0.50 \\
V10 & 0.92 & 0.92 & 0.25 \\
V16 & 0.98 & 0.98 & 0.95 \\
\hline
\end{tabular}

\section{LIMITATIONS}

It is noteworthy that this study was meant to be carried out in a macroscopic level. In other words, countries were considered in their entirety as opposed to looking into each individual city of a given country. It is known that each city within a given country in this study will have different meteorological data from the average of the two main cities considered. However, reported number of cases in the literature showed that the majority of the cases (sometimes up to $95 \%$ ) is within a specific geographical region of the country under consideration as opposed to being equally spread across the country's geographical areas. As an example, in Italy the region between the latitudes $41 \mathrm{~N}$ (Milan) and $45 \mathrm{~N}$ (Rome) parallels reported around 90\% of the cases with variation in temperature and humidity of $10-15 \%$ in the reported averages ${ }^{28}$. These ranges are acceptable in the approximate analysis presented in this study.

\section{CONCLUSION}

We presented a comprehensive study of factors affecting the spread of the new coronavirus in seven of the most affected countries by COVID-19 during the first wave. We used reported data in various published and publically available websites including those published by the World Odometer. We proposed an inductive formula that contains some factors that are believed to affect the coronavirus reported daily cases in those countries. The factors used in this formula were five: the temperature, the humidity, the lockdown times, the number of COVID-19 tests performed by a given country and the expenditure. Data were collected in the period from March 15 till June 30, coinciding with the peak of the virus spread in the countries under consideration. The results produced two groups of countries corresponding to different sets of possible $\mathrm{K}$ values. The first group of countries included Germany, France, South Korea, Spain and Italy with corresponding $\mathrm{K}$-values of $\mathrm{K}_{1}=26 \%, \mathrm{~K}_{2}=32 \%$ and $\mathrm{K}_{5}=39 \%$. The second group of countries included Canada and the UK where the most effective factors were the humidity, the expenditure, and the number of COVID-19 tests, with corresponding values of $\mathrm{K}_{2}=35 \%, \mathrm{~K}_{3}=26 \%$ and $\mathrm{K}_{5}=28 \%$. The produced results suggested that in the first group of countries the government measure of extensive curfew significantly contributed to the reduction of daily reported cases (such as Italy and Spain), while 
in the second group of countries, the expenditure has contributed positively to the reduction of the reported daily cases of COVID-19, for instance, in Canada.

\section{FUTURE STUDIES}

Despite having tested our approach on a sample composed of seven countries, the approach can be generalized to include many other countries, as well as other factors such as the population density, the UV level and wind effects. We are also assessing the effect of the aforementioned parameters during the second wave of COVID-19. Our approach can also be used to predict future daily cases in a given country, since an advance knowledge of the weather conditions in a given country at a given time/ week/season is available in addition to projected curfew hours. Alternatively, this approach can propose curfew hours that are sufficient enough to "flatten the curve" and to keep the daily cases to a level that healthcare infrastructure can handle.

\section{ACKNOWLEDGMENTS}

This research is supported by the COVID-19 special fund $\mathrm{N}^{\circ} 14 / 41$ from the Islamic University of Madinah, Saudi Arabia. The authors would like to acknowledge the support of King Fahd University of Petroleum and Minerals for this research.

\section{REFERENCES}

1. The World Odometer. COVID-19 Coronavirus pandemic. [cited 2021 May 14]. Available from: https://www.worldometers. info/coronavirus/\#countries

2. de Courten M, Pogrmilovic B, Apostolopoulos V. Europe's second wave is worse than the first: what went so wrong, and what can it learn from countries like Vietnam? [cited 2021 May 14] Available from: https://theconversation.com/europes-secondwave-is-worse-than-the-first-what-went-so-wrong-and-whatcanit-learn-from-countries-like-vietnam-147907

3. World Health Organization. Coronavirus disease (COVID-19) pandemic. [cited 2021 May 14]. Available from: https://www. who.int/emergencies/diseases/novel-coronavirus-2019

4. Orlik T, Rush J, Cousin M, Hong J. Coronavirus could cost the global economy \$2.7 trillion: here's how. [cited 2021 May 14]. Available from: https://www.bloomberg.com/graphics/2020coronavirus-pandemic-global-economic-risk/

5. Chowell G, Sattenspiel L, Bansal S, Viboud C. Mathematical models to characterize early epidemic growth: a review. Phys Life Rev. 2016;18:66-97.

6. World Health Organization. WHO Coronavirus (COVID-19) dashboard. [cited 2021 May 14] Available from: https:// covid19.who.int/

7. Centers for Disease Control and Prevention. COVID data tracker. [cited 2021 May 14]. Available from: https://covid.cdc.gov/ covid-data-tracker/\#cases casesper100klast7days

8. Pica N, Bouvier NM. Environmental factors affecting the transmission of respiratory viruses. Curr Opin Virol. 2012;2:90-5.

9. Rashed EA, Kodera S, Gomez-Tames J, Hirata A. Influence of absolute humidity, temperature and population density on COVID-19 spread and decay durations: multi-prefecture study in Japan. Int J Environ Res Public Health. 2020;17:5354.

10. Şahin M. Impact of weather on COVID-19 pandemic in Turkey. Sci Total Environ. 2020;728:138810.

11. Tantrakarnapa K, Bhopdhornangkul B, Nakhaapakorn K. Influencing factors of COVID-19 spreading: a case study of Thailand. J Public Health. 2020 In Press.

12. Tariq A, Roosa K, Mizumoto K, Chowell G. Assessing reporting delays and the effective reproduction number: the Ebola epidemic in DRC, May 2018-January 2019. Epidemics. 2019;26:128-33.

13. Nishiura H, Chowell G. Early transmission dynamics of Ebola virus disease (EVD), West Africa, March to August 2014. Euro Surveill. 2014;19:20894.

14. Jia L, Li K, Jiang Y, Guo X, Zhao T. Prediction and analysis of Coronavirus disease 2019. arXiv. In Press 2020.

15. Liang K. Mathematical model of infection kinetics and its analysis for COVID-19, SARS and MERS. Infect Genet Evol. 2020;82:104306.

16. Li Q, Guan X, Wu P, Wang X, Zhou L, Tong Y, et al. Early transmission dynamics in Wuhan, China, of novel coronavirusinfected pneumonia. N Engl J Med. 2020;382:1199-207.

17. Shim E, Tariq A, Choi W, Lee Y, Chowell G. Transmission potential and severity of COVID-19 in South Korea. Int J Infect Dis. 2020;93:339-44.

18. D'Arienzo M, Coniglio A. Assessment of the SARS-CoV-2 basic reproduction number, $\mathrm{R} 0$, based on the early phase of COVID-19 outbreak in Italy. Biosaf Health. 2020; 2:57-9.

19. Nioi M, Napoli PE, Lobina J, Fossarello M, d'Aloja E. COVID-19 and Italian healthcare workers from the initial sacrifice to the mRNA vaccine: pandemic chrono-history, epidemiological data, ethical dilemmas, and future challenges. Front Public Health. 2020;8:591900.

20. Huang R, Liu M, Ding Y. Spatial-temporal distribution of COVID-19 in China and its prediction: a data-driven modeling analysis. J Infect Dev Ctries. 2020;14:246-53.

21. Napoli PE, Nioi M. Global spread of coronavirus disease 2019 and malaria: an epidemiological paradox in the early stage of a pandemic. J Clin Med. 2020;9:1138.

22. Giuliani D, Dickson MM, Espa G, Santi F. Modelling and 
predicting the spatio-temporal spread of COVID-19 in Italy. BMC Infect Dis. 2020;20:700.

23. Briz-Redón A, Serrano-Aroca A. A spatio-temporal analysis for exploring the effect of temperature on COVID-19 early evolution in Spain. Sci Total Environ. 2020;728:138811.

24. Lakshmi Priyadarsini S, Suresh M. Factors influencing the epidemiological characteristics of pandemic COVID 19: a TISM approach. Int J Healthc Manag. 2020;13:89-98.

25. Casella F. Can the COVID-19 epidemic be controlled on the basis of daily test reports?. IEEE Control Syst Lett. 2021;5:1079-84.

26. Wong CK, Wong JY, Tang EH, Au CH, Wai AK. Impact of national containment measures on decelerating the increase in daily new cases of COVID-19 in 54 countries and 4 epicenters of the pandemic: comparative observational study. J Med Internet Res. 2020;22:e19904.

27. Our World in Data. Statistics and research: Coronavirus pandemic (COVID-19). [cited 2021 May 14]. Available from: https:// ourworldindata.org/coronavirus\#coronavirus-country-profiles

28. Current Results. [cited 2021 May 14]. Available from: https:// www.currentresults.com/index.php

29. Anderson J, Bergamini E, Brekelmans S, Cameron A, Darvas Z, Domínguez Jíménez M, et al. The fiscal response to the economic fallout from the coronavirus. [cited 2021 May 14]. Available from: https://www.bruegel.org/publications/datasets/ covid-national-dataset/ 